\title{
El extracto leucocitario dializable aumenta la concentración de interferón gamma sérico en cerdos destetados ${ }^{\#}$
}

\author{
Dialyzable leukocyte extract as inductor of interferon gamma concentration \\ in serum of weaned pigs
}

\author{
P Hernández-Peralta ${ }^{a}$, SM Pérez-Tapia ${ }^{b}$, AY Limón-Flores ${ }^{b}$, S Vázquez-Leyva ${ }^{b}$, S Estrada-Parra ${ }^{b}$, \\ I Sánchez-Betancourt ${ }^{c}$, JA Navarro-Hernández ${ }^{\mathrm{d}}$, L Cobos-Marín ${ }^{\mathrm{*}}$ \\ a Departamento de Microbiología e Inmunología, Facultad de Medicina Veterinaria y Zootecnia, Universidad Nacional Autónoma de \\ México, México D.F., México. \\ bepartamento de Inmunología, Escuela Nacional de Ciencias Biológicas, IPN, Colonia Santo Tomas, México D.F., México. \\ 'Departamento de Producción: cerdos, Facultad de medicina veterinaria y Zootecnia, Universidad Nacional Autónoma de México, \\ México D.F., México. \\ dDepartamento de Genética y Bioestadística, Facultad de Medicina Veterinaria y Zootecnia, \\ Universidad Nacional Autónoma de México, México D.F., México.
}

\begin{abstract}
SUMMARY
In most intensive pig production systems piglets are weaned at 3 weeks of age, which creates stress because of feeding changes and hierarchy. This increases the incidence of respiratory symptoms, diarrhea, poor weight gain and cause economic losses. Dialyzable leukocyte extract (DLE) can enhance the immune response by increasing the levels of interferon gamma (IFN- $\gamma$ ). In this study we evaluated the effect of porcine DLE (DLEp), when applied 7 times intradermally in piglets during the first four weeks at two different doses. Forty eight piglets were divided into 3 groups of 16 animals each. Group A: high dose $(77 \mathrm{mg} / \mathrm{kg}$ ), group B: low dose $(17 \mathrm{mg} / \mathrm{kg})$, group C: control (sodium chloride). Blood and serum samples were obtained at days 0,14 and 30 for leukocyte accounts by haemogram and IFN- $\gamma$ quantification by ELISA. In addition, we determined the daily incidence of diarrhea and respiratory symptoms and measured the weight of the animals studied. The high dose $(77 \mu \mathrm{g} / \mathrm{kg}), \mathrm{produced}$ a significant increase in serum IFN- $\gamma$ concentration in the third sampling $(\mathrm{d}=2,288, \alpha=0.05, \mathrm{P}=0.003)$. The rest of the variables analyzed showed no significant difference between the treated and the control groups $(\mathrm{P}>0.05)$. Conclusion: applying seven DLEP doses intradermally in weaned pigs significantly increased IFN- $\gamma$ concentration in the serum at 30 days post treatment.
\end{abstract}

Key words: dialyzable leukocyte extract, gamma interferon, piglets.

\section{RESUMEN}

En la mayoría de los sistemas intensivos de producción porcina se desteta a los lechones a las 3 semanas de edad, lo que genera estrés por el cambio de alimentación y jerarquización. Esto aumenta la incidencia de cuadros respiratorios, diarreas, baja las ganancias de peso y causa pérdidas económicas. El extracto leucocitario dializable (DLE) puede mejorar la respuesta inmune al aumentar los niveles de IFN- $\gamma$. En el presente trabajo se valoró el efecto del DLE porcino (DLEp) al ser aplicado 7 veces por vía intradérmica en lechones durante las primeras cuatro semanas del destete a dos dosis diferentes. Se utilizaron 48 lechones divididos en 3 grupos de 16 animales. Grupo A: dosis alta (77 $\mu \mathrm{g} / \mathrm{kg}$ ), grupo B: dosis baja (17 $\mu \mathrm{g} / \mathrm{kg}$ ), grupo C: testigos (solución salina). Se obtuvieron muestras de sangre completa y suero a los días 0,14 y 30 para realizar cuentas leucocitarias por hemograma y la cuantificación de IFN- $\gamma$ por ELISA. Además se determinó la incidencia diaria de diarreas y cuadros respiratorios y se midió el peso de todos los animales estudiados. La dosis alta $(77 \mu \mathrm{g} / \mathrm{kg})$, produjo un aumento significativo en la concentración de IFN- $\gamma$ sérico en el tercer muestreo $(\mathrm{d}=2,288, \alpha=0,05, \mathrm{P}=0,003)$. El resto de las variables analizadas no mostraron diferencia significativa entre los grupos tratados y el testigo $(P>0,05)$.

Palabras clave: extracto leucocitario dializable, interferón gamma, lechones.

\section{INTRODUCCIÓN}

La mayoría de los sistemas intensivos de crianza de cerdos en el mundo destetan a los lechones a las 3 semanas de edad. Lo anterior, sumado al estrés por el cambio de alimentación y jerarquización, aumenta la incidencia de

Aceptado: 26.12.2013.

\# Proyecto Factor de Transferencia ENCB, IPN: IB-11-07.

* laura.cobosmarin@gmail.com cuadros respiratorios y diarreas, disminuye las ganancias de peso y causa pérdidas económicas. Esta condición puede presentarse incluso en granjas con poca incidencia de enfermedades, debido generalmente a la presencia de patógenos oportunistas.

El empleo de inmunomoduladores en esta etapa del desarrollo podría coadyuvar a eliminar la incidencia de estos cuadros al mejorar la respuesta inmune de los animales. Un ejemplo de esto es el uso de citocinas que estimulan la activación de linfocitos para modular la respuesta inmune 
celular (RIC) (Kogut y col 2002, Min y col 2001); particularmente el IFN- $\gamma$ se ha aplicado en la vacunación contra algunos agentes intracelulares (Rabinovich y col 1994). Sin embargo, el costo y la disponibilidad de citocinas comerciales hacen difícil su utilización, por lo que al ser de bajo costo, el extracto dializable leucocitario o DLE (por sus siglas en inglés: Dialyzable Leukocyte Extracts) podría emplearse como una alternativa para inducir una inmunomodulación.

El DLE, anteriormente conocido como Factor de Transferencia, fue descrito por primera vez por Sherwood Lawrence (Lawrence 1955). Está formado por un conjunto de péptidos lábiles al calor, pero muy estables al frío, que pueden almacenarse por varios años a temperaturas entre $-20{ }^{\circ} \mathrm{C}$ y $-70{ }^{\circ} \mathrm{C}$ (Estrada-Parra y col 1983). El mecanismo por donde el DLE ejerce su efecto biológico no ha sido completamente establecido, los primeros estudios en humanos mostraron que inhibe la migración de macrófagos de una manera antígeno específica (Wilson y Funderberg 1981), transfiere la respuesta de intradermorreacción antígeno específica, aumenta la proliferación y actividad de linfocitos T (Kirkpatrik y col 1972), induce la producción de citocinas en respuesta a antígenos específicos y aumenta la resistencia a infecciones en inmunodeficiencias de carácter genético (Spitler y col 1972). Estudios más recientes en ratones han demostrado que el DLE induce la producción de citocinas como la IL2 y el IFN- $\gamma$ (Levin y col 1975) (Álvarez-Thull y Kirkpatrik 1996).

Diversos trabajos han demostrado que el DLE puede tener efecto interespecies, por ejemplo: el bovino se ha utilizado en aves (Wilson 1988) y el porcino se ha aplicado a un modelo de resistencia a Listeria en ratón. El DLE ha sido ampliamente usado en humanos en la protección y el control de enfermedades infecciosas virales (Padierna y col 1985, Pizza y col 1996, Steele y col 1980), micobacterianas (Bullock y col 1972, Fabre y col 2004) y parasitarias (Delgado y col 1981). En medicina veterinaria ha sido utilizado en la prevención de algunas enfermedades en suinos (Rojas 1987), aves (Klesius y col 1984, Bravo-Blas y col 2010) y bovinos (Mateos 1992).

El objetivo del presente trabajo fue demostrar que la aplicación intradérmica del extracto leucocitario dializable porcino (DLEp) en cerdos, durante las primeras cuatro semanas postdestete, puede aumentar las cuentas leucocitarias en sangre periférica además de modular la respuesta inmune hacia un perfil Th1 al incrementar los niveles de interferón gamma (IFN- $\gamma$ ). Cabe destacar que este es un estudio piloto, en el que se buscó encontrar la dosis óptima a emplear en esta etapa de la producción.

\section{MATERIAL Y MÉTODOS}

\section{EXTRACTO LEUCOCITARIO DIALIZABLE PORCINO (DLEP)}

Obtención del bazo. El DLEp fue obtenido a partir del bazo de una cerda adulta clínicamente sana, de tercer parto, de
$173 \mathrm{~kg}$ de peso, destinada al sacrificio mediante choque eléctrico y desangrado, de acuerdo con la norma oficial mexicana: NOM-033-ZOO-1995 (Secretaría de Agricultura, Ganadería y Desarrollo Rural. 1995) en el centro de enseñanza, investigación y extensión en producción porcina (CEIEPP) FMVZ, UNAM. La obtención del órgano se llevó a cabo en el espacio destinado para el faenado, con la previa desinfección del área con hipoclorito de sodio al 1\%. El material para realizar la incisión, así como el recipiente donde se colocó el órgano, se encontraban estériles.

El bazo obtenido pesó 310 gramos y se sometió a un proceso de extracción del parénquima, para ello se quemó la cápsula y capa superficial del tejido con una espátula calentada en un mechero, con la finalidad de evitar al máximo la contaminación microbiana. Se desechó el tejido quemado y se obtuvieron 151 gramos de parénquima que se colocaron en solución salina isotónica de cloruro de sodio al $0,9 \%$ estéril y se congelaron a $-20{ }^{\circ} \mathrm{C}$, en un vaso de precipitados previamente horneado a $300{ }^{\circ} \mathrm{C}$ por dos horas.

Dializado. El parénquima extraído del bazo se maceró con una malla de aluminio con $30 \mathrm{~mL}$ de solución salina isotónica de cloruro de sodio al 0,9\%. El macerado se sometió a 10 ciclos de congelación-descongelación y se dializó, por centrifugación, a 5.000 gravedades, en tubos Amicon® ultra con corte de $10 \mathrm{kDa}$. El dializado se esterilizó por filtración en membrana de 0,22 micras y se almacenó en frascos de $3 \mathrm{~mL}$. Posteriormente se hizo la determinación de proteínas utilizando un estuche comercial por el micrométodo del ácido bicinconínico (BCA) (Pierce $^{T M}$ ) siguiendo las instrucciones del fabricante $\mathrm{y}$ se procedió a envasar los extractos a una concentración de $500 \mu \mathrm{g} / 2 \mathrm{ml}$.

Control de calidad. Se determinó que el producto se encontraba libre de endotoxinas bacterianas por el método de lisado de amebocitos de Limulus (LAL) (Gould 1998). Se realizó un barrido espectrofotométrico UV, en un intervalo de 200 a $350 \mathrm{~nm}$, para conocer la huella espectral del DLEp. Esta obtuvo un pico máximo de absorbancia entre los 240 y $260 \mathrm{~nm}$ (dato no mostrado).

Liofilización. Los frascos se congelaron a $-78{ }^{\circ} \mathrm{C}$ durante 24 horas, después se trasladaron en hielo seco a la liofilizadora Labconco Free Zone 2.5 donde se liofilizaron a $-0,5$ atmósferas durante 16 horas. Se obtuvieron frascos con una concentración de 500 microgramos de DLEp. Estos se reconstituyeron en $0,5 \mathrm{~mL}$ de solución salina isotónica de cloruro de sodio al 0,9\% para su aplicación.

\section{GRUPOS DE ESTUDIOY TRATAMIENTOS}

Para el estudio se utilizaron 48 lechones York-Landrace de 21 días de edad provenientes de 4 camadas de cerdas York-Landrace de la granja, que fueron aleatoriamente 
distribuidos en 3 grupos de 16 animales. Grupo A: dosis alta $(77 \mu \mathrm{g} / \mathrm{kg})$, grupo B: dosis baja $(17 \mu \mathrm{g} / \mathrm{kg})$, grupo C: testigos (solución salina isotónica de cloruro de sodio). El DLEp se aplicó 7 veces por vía intradérmica, dividido en dos etapas: las 5 primeras cada 3 días y las últimas 2 cada 8 días.

Características de la producción en la granja. La granja es positiva a influenza porcina, parvovirus porcino, erisipela, rinitis atrófica y ascaris suum. Las vacunas que se emplean en las cerdas son: influenza porcina 18-20 días después del servicio y la vacuna triple (parvovirus porcino, leptospira y erisipela) 15 días antes del parto.

En la granja se cuenta con un sistema de corraletas elevadas de $5,25 \mathrm{~m}^{2}$ teniendo $0,437 \mathrm{~m}^{2}$ por animal. Se colocó cada camada en una corraleta distinta, el área de destete cuenta con calentadores de gas para mantener la temperatura de la caseta en $27^{\circ} \mathrm{C}$. Durante el periodo del estudio los lechones recibieron alimento comercial iniciador etapa 2 marca Nupig®, tres veces al día.

Toma de muestras. Se realizaron 3 muestreos sanguíneos de $7 \mathrm{~mL}$ con la técnica de la vena cava anterior: el día 0,14 y 30 , de los que se obtuvieron muestras de sangre con anticoagulante para realizar cuentas leucocitarias por hemograma y muestras de sangre sin anticoagulante para obtener suero y cuantificar IFN- $\gamma$, por ELISA.

\section{EVALUACIÓN DE PARÁMETROS INMUNOLÓGICOS}

A las muestras sanguíneas con anticoagulante se les realizó el conteo y determinación de poblaciones leucocitarias: plaquetas y leucocitos totales. El suero obtenido de los tubos sin anticoagulante se utilizó para detectar la concentración de IFN- $\gamma$ mediante pruebas de ELISA de captura con el estuche comercial: invitrogen C\# KSC4022, siguiendo las instrucciones del fabricante.

\section{EVALUACIÓN DEL ESTADO CLÍNICO Y PESO}

El estado clínico fue evaluado diariamente durante el tiempo del estudio, para poder determinar la incidencia de fiebre, cuadros respiratorios (presencia de tos, respiración abdominal y exudado nasal) y diarrea (observación perianal y estimulación en la región lumbar). El peso corporal individual de los animales fue medido los lunes durante cinco semanas.

\section{ANÁLISIS ESTADÍSTICO}

Para las variables cuantitativas se empleó la prueba de bondad de ajuste de Shapiro-Wilk para determinar normalidad, en compañía de la función Box-Cox para su normalización. En las que se distribuyeron normalmente el análisis descriptivo incluyó estimadores de número de observaciones, promedios, error estándar de la media e intervalo de confianza de $95 \%$ para la media. En las que se tuvieron varias mediciones en el tiempo, se hizo un análisis de varianza multivariado (MANOVA), considerando como criterio de decisión estadística la lambda de Wilks $(\lambda)$ $(\alpha=0,05)$. y análisis de varianza (ANOVA) con la prueba de comparación de promedios de Dunnett $(\alpha=0,05)$ cuando hubo solo una medición en el tiempo. En las variables que no se distribuyeron normalmente los estimadores descriptivos fueron: número de observaciones, el valor mínimo, máximo y mediana. Estas fueron analizadas por medio de contraste no paramétrico de la suma de rangos Willcoxon/ Kruskal/Wallis Ji cuadrada $\left(\mathrm{x}^{2}\right)(\alpha=0,05)$.

Para las variables cualitativas se utilizaron tablas de contingencia con la prueba de $\mathrm{x}^{2}$ de Pearson para determinar la posible relación entre el acumulado en la incidencia de casos y tratamientos.

\section{RESULTADOS}

\section{EVALUACIÓN DE PARÁMETROS INMUNOLÓGICOS}

Interferón gamma. Los valores correspondientes a los muestreos 1 y 2 fueron inferiores a los que puede detectar la prueba, por lo que no se incluyeron en el cuadro de estadística descriptiva.

La dosis alta $(77 \mu \mathrm{g} / \mathrm{kg})$ produjo un aumento significativo en la concentración de IFN- $\gamma$ sérico en el tercer muestreo $(\mathrm{d}=2,288, \alpha=0,05, \mathrm{P}=0,003)$ (cuadro 1$)$. El resto de las variables analizadas no mostraron diferencia significativa entre los grupos tratados y el testigo $(\mathrm{P}>0,05)$.

Cuadro 1. Estadística descriptiva de la concentración de interferón gamma en sangre periférica de cerdos York- Landrace tratados con dos dosis de DLEp.

Descriptive statistics of interferon gamma concentration in peripheral blood of York-Landrace pigs treated with two doses of DLEp.

\begin{tabular}{lccccc}
\hline \multicolumn{1}{c}{ Tratamiento } & Muestreo & $\mathrm{n}$ & Promedio $\mathrm{pg} / \mathrm{mL}$ & EEM & IC 95\% \\
\hline Dosis alta & 3 & 14 & 437,15 & 45,19 & $534,8-339,5$ \\
Dosis baja & 3 & 16 & 208,84 & 47,05 & $309,1-108,5$ \\
Grupo testigo & 3 & 15 & 223,65 & 36,45 & $301,8-145,4$ \\
\hline
\end{tabular}

n: tamaño de la muestra, EEM: Error estándar de la media, IC 95\%: Intervalo de confianza del 95\% de la media, pg/mL. n: sample size, EEM: Standard error of the mean, IF 95\%: Confidence interval 95\% of the mean pg/mL. 
Cuentas leucocitarias y plaquetarias. Las variables de cuentas plaquetarias y leucocitos totales no mostraron una diferencia significativa entre los grupos tratados y el testigo. Plaquetas $\left(\mathrm{F}_{2,60}=0,78, \mathrm{P}=0,3758\right)$, leucocitos totales $\left(\mathrm{F}_{2,89^{\circ}}=0,117, \mathrm{P}=0,899\right)$ (cuadros 2 y 3 ).

\section{EVALUACIÓN DEL ESTADO CLÍNICO Y PESO}

En la figura 1 se muestra el número de animales que presentaron fiebre, diarreas y cuadros respiratorios a lo largo del experimento. No hubo diferencias significativas en ninguno de los parámetros evaluados: fiebre $\left(\mathrm{R}^{2}=0,0341, \mathrm{X}_{2}^{2}=2,133, \mathrm{P}=0,3442\right)$; diarreas $\left(\mathrm{R} 2=0,0115, \mathrm{X}_{2}{ }_{2}=0,729, \mathrm{P}=0,6947\right)$ y signología respiratoria $\left(\mathrm{R}^{2}=0,0098, X_{2}{ }_{2}=0,582, \mathrm{P}=0,747\right)$. El análisis del peso corporal no mostró diferencia significativa debida a la interacción tratamiento ganancia de peso $\left(\mathrm{F}_{2,44}=0,8792, \mathrm{P}=0,4223\right)$ (figura 2).

\section{DISCUSIÓN}

Diferentes estudios demuestran que el DLE estimula una inmunomodulación hacia un perfil de tipo Th1 (Calzada 2000, Ortiz y col 2006, Bravo-Blas y col 2010). Los resultados del presente estudio concuerdan con esos hallazgos, ya que se observó una modulación significativa de IFN- $\gamma$ en el suero sanguíneo de los cerdos que recibieron una dosis alta de DLE.

El IFN- $\gamma$ es una citocina que participa tanto en la respuesta inmune innata como adaptativa. Juega un papel importante en la infección por patógenos intracelulares, ya que favorece en perfil Th1 y de esta manera participa en la activación de células NK, neutrófilos, macrófagos y linfocitos TCD8+ (Samuel 2001). También estimula la producción de anticuerpos y el cambio de isotipo a IgG2 (Yilma y col 1989, Stark y col 1998, McCormick y col 2001, Schijns y col 2002, Snapper y Paul 1987, De Wit y col 2004).

Cuadro 2. Cuentas plaquetarias en sangre periférica de cerdos York-Landrace tratados con dos dosis de DLEp. Peripheral blood platelet counts in York-Landrace pigs treated with two doses of DLEp.

\begin{tabular}{lccccc}
\hline Tratamiento & Muestreo & $\mathrm{n}$ & Promedio $10^{9} \mathrm{~L}^{-1}$ & EEM & IC 95\% \\
\hline \multirow{3}{*}{ Dosis alta } & 1 & 16 & 573,81 & 42,46 & $664,32-483,30$ \\
& 2 & 7 & 466,42 & 35,44 & $553,16-379,69$ \\
& 3 & 15 & 526,93 & 24,63 & $579,76-474,09$ \\
Dosis baja & 1 & 10 & 527,4 & 48,82 & $637,85-416,94$ \\
& 2 & 7 & 455,57 & 51,29 & $581,09-330,05$ \\
Grupo testigo & 3 & 15 & 555,13 & 37,17 & $534,7-375,39$ \\
& 1 & 76 & 443,68 & 33,56 & $615,23-472,14$ \\
& 2 & 7 & 442,08 & 39,56 & $548,27-347,72$ \\
\end{tabular}

n:tamaño de la muestra, EEM: error estandar de la media, IC 95\%: intervalo de confianza a $95 \%$ de la media. $\mathrm{n}$ : sample size, EEM: Standard error of the mean, IF 95\%: Confidence interval 95\% of the mean.

Cuadro 3. Leucocitos totales en sangre periférica de cerdos York-Landrace tratados con dosis de DLEp. Peripheral blood total leucocytes in York-Landrace pigs treated with two doses of DLEp.

\begin{tabular}{|c|c|c|c|c|c|c|}
\hline Tratamiento & Muestreo & $\mathrm{n}$ & $\begin{array}{c}\text { Promedio } \\
10^{9} \mathrm{~L}^{-1}\end{array}$ & EEM & IC $95 \%$ & Transformación Box-Cox \\
\hline \multirow{3}{*}{ Dosis alta } & 1 & 16 & 9,94 & 0,658 & $11,34-8,54$ & $\left.\left(\left(“ 1^{\circ} \mathrm{M} \text { ") }\right)^{-0.8}\right)-1\right) /-0,0152$ \\
\hline & 2 & 16 & 14,31 & 0,747 & $15,90-12,71$ & $\log \left(\right.$ (“2 $\left.\left.2^{\circ} \mathrm{M} "\right)\right) * 14,3854$ \\
\hline & 3 & 16 & 17,56 & 0,793 & $19,26-15,87$ & $\left.\left((\text { (“3० M") })^{-0.4}\right)-1\right) /-0,006$ \\
\hline \multirow{3}{*}{ Dosis baja } & 1 & 16 & 8,36 & 0,442 & $9,30-7,42$ & $\left.\left(\left(\text { (“ } 1^{\circ} \mathrm{M} \text { ") }\right)^{-0.8}\right)-1\right) /-0,0152$ \\
\hline & 2 & 16 & 15,11 & 0,664 & $16,42-13,70$ & $\log \left(\right.$ (“2 $\left.\left.2^{\circ} \mathrm{M} "\right)\right) * 14,3854$ \\
\hline & 3 & 15 & 20,39 & 1,359 & $23,30-17,47$ & $\left.\left(\left(“ 3^{\circ} \mathrm{M}^{\prime \prime}\right)^{-0.4}\right)-1\right) /-0,006$ \\
\hline \multirow{3}{*}{ Grupo testigo } & 1 & 16 & 9,62 & 0,692 & $11,10-8,14$ & $\left.\left(\left(“ 1^{\circ} \mathrm{M}^{\prime}\right)^{-0.8}\right)-1\right) /-0,0152$ \\
\hline & 2 & 15 & 14,60 & 0,915 & $16,56-12,64$ & $\log \left(\left(“ 2^{\circ} \mathrm{M} ”\right)\right) * 14,3854$ \\
\hline & 3 & 14 & 18,02 & 1,147 & $20,50-15,54$ & $\left.\left(\left(\text { (“3 }{ }^{\circ} \mathrm{M}^{\prime}\right)^{-0.4}\right)-1\right) /-0,006$ \\
\hline
\end{tabular}

n:tamaño de la muestra, EEM: error estandar de la media, IC 95\%: intervalo de confianza a 95\% de la media, Box-Cox: función para la transformación de la variable a la normalidad.

n: sample size, EEM: Standard error of the mean, IF 95\%: Confidence interval 95\% of the mean, Box-Cox: Function to transform the variable to normal. 


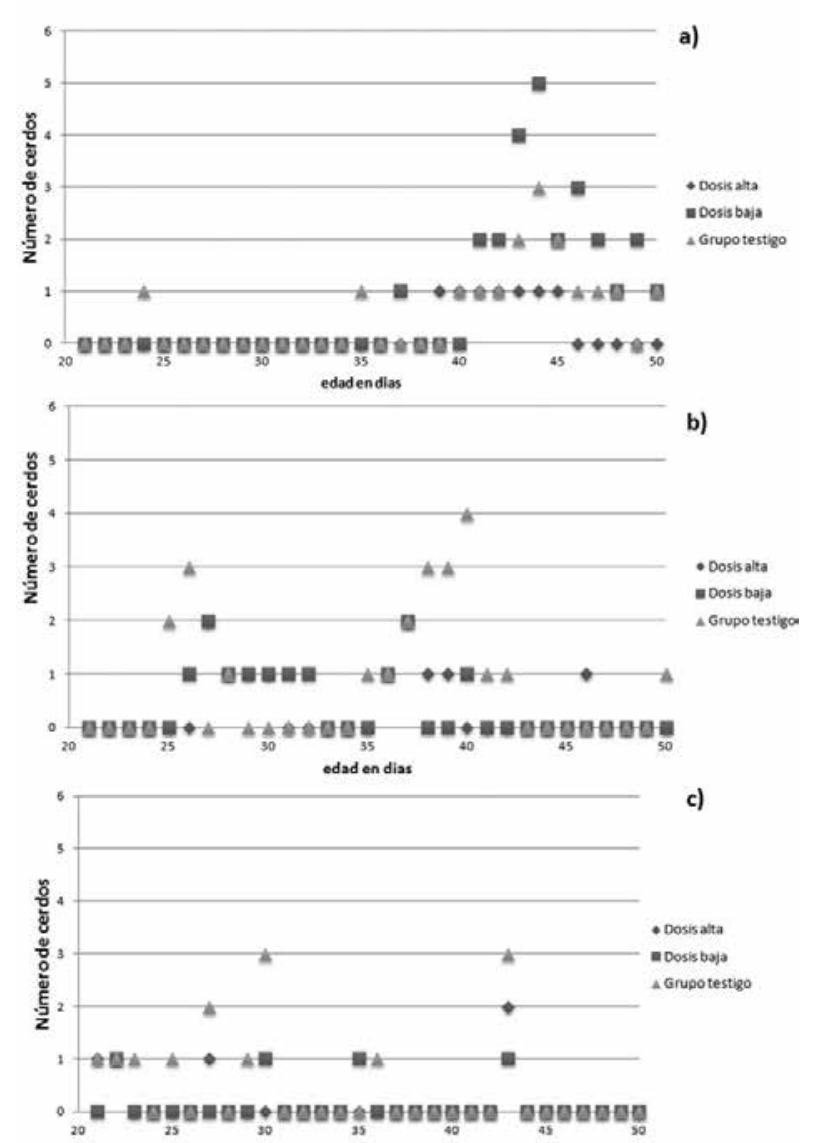

Figura 1. Incidencia de signos clínicos por edad (días) en cerdos York-Landrace tratados con dos dosis de DLEp. a) cuadros respiratorios, b) diarrea, c) fiebre.

Incidence of clinical signs by age (days), in York-Landrace pigs treated with two doses of DLEp. a) respiratory symptoms, b) diarrhea, c) fever.

Analizando los resultados de incidencias de signos, observamos que el aumento de IFN- $\gamma$ se presenta tras registrarse la presencia de signos respiratorios en todos los grupos, desde el día 40 de edad y hasta el término del estudio (figura 1a). Aun cuando el incremento en los signos respiratorios no es significativo respecto del tratamiento $(\mathrm{P}=0,747)$, podría sugerir que en este período del experimento, en el que la inmunidad pasiva disminuye, los animales estuvieron expuestos a ciertos microorganismos circulantes en la granja. Lo anterior podría ser un factor determinante en la síntesis de INF $\gamma$, ya que la estimulación antigénica en conjunto con el extracto leucocitario dializable puede inducir una respuesta de tipo Th1 (Ávalos 2008, Bravo-Blas y col 2010, Fabre y col 2004).

Se ha encontrado que el DLE posee la característica de estimular TLR 2 (Robledo-Ávila y col 2006). Este receptor puede activarse por diversos componentes microbianos y desencadenar la síntesis de citocinas entre las que destacan: IL1, IL6, TNF $\alpha$ e IL12. Esta última tiene la capacidad de estimular a las células NK y linfocitos Th1

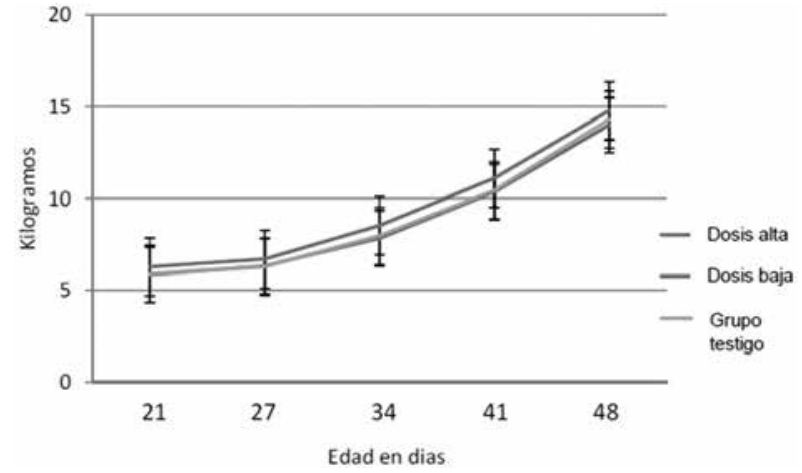

Figura 2. Promedio de peso corporal en cerdos York-Landrace tratados con dos dosis de DLEp. Las barras verticales corresponden a un error estándar de la media.

Average body weight in York-Landrace pigs treated with two doses of DLEp. Vertical bars correspond to standard error of the mean.

para la producción de IFN- $\gamma$ (Kohno 1997). Por lo tanto es posible pensar que el DLEp utilizado en este trabajo tuvo la capacidad de estimular la producción de IFN- $\gamma$ por la vía de TLRs; sin embargo, habría que hacer ensayos que demostraran esta hipótesis.

En este estudio no se observaron diferencias en cuanto a la presencia de fiebre, signos respiratorios, digestivos o ganancia de peso. Lo anterior pudo deberse a que el trabajo se realizó en un granja con buenas prácticas de bioseguridad y pocos problemas infecciosos. La inducción de una respuesta inmune de tipo Th1 podría ser relevante en granjas donde la presencia de enfermedades infecciosas sea alta, y el aumento significativo del IFN- $\gamma$ pudiera jugar un papel relevante en el control de dichas enfermedades. Actualmente se están llevando a cabo estudios con la aplicación del DLEp en granjas con presencia de Síndrome Reproductivo y Respiratorio Porcino (PRRS), para demostrar que un aumento de esta citocina favorece el control de la enfermedad y evita pérdidas económicas por disminución en la ganancia de peso.

Los resultados del presente estudio, en el que se evaluó la respuesta inmunológica de cerdos destetados de 21 a 50 días de edad, tratados con siete dosis de extractos dializables leucocitarios porcinos por vía intradérmica, demostraron que la dosis alta $(77 \mu \mathrm{g} / \mathrm{kg})$ aumentó significativamente la concentración de IFN- $\gamma$ sérico respecto del grupo testigo.

\section{AGRADECIMIENTOS}

Los autores agradecen al MVZ Roberto Martínez, MVZ Mónica Sánchez, M en C. Luis Enrique García y M en C. Ana Rodríguez por su apoyo en la realización de este trabajo.

\section{REFERENCIAS}

Ávalos H. 2008. Obtención y caracterización de extractos dializables leucocitarios de origen bovino. Tesis de maestría, Escuela Nacional de Ciencias Biológicas, Instituto Politécnico Nacional, México DF, México. 
Álvarez-Thull L, CH Kirkpatrick. 1996. Profiles of cytokine production in recipients of transfer factors. Biotherapy 9, 55-59.

Bullock WE, JP Fields, MW Brandriss. 1972. An evaluation of transfer factor as immunotherapy for patients with lepromatous leprosy. N Engl J Med 287, 1053-1059.

Bravo-Blas A, R Téllez, S Uribe, F Salmerón, L Valdés, S Estrada-Parra, L Cobos-Marín. 2010. El factor de transferencia como inductor de la expresión de IFN- $\gamma$ e IL-2 en pollos vacunados contra influenza aviar. Arch Med Vet 42, 67-71.

Calzada NG. 2000. Uso en lechones de factor de transferencia y parapoxvirus inactivado (Baypamun) en la vacunación contra el virus de Aujeszky. Tesis de maestría, Escuela Nacional de Ciencias Biológicas, Instituto Politécnico Nacional, México DF, México.

Delgado O, EL Romano, E Belfort, F Pifano, JV Scorza, Z Rojas. 1981. Dialyzable leukocyte extract therapy in immunodepressed patients with cutaneous leishmaniasis. Clin Immunol Immunopathol 19, 351-359.

De Wit MC, C Horzinek, L Haagmans, CJ Schijns. 2004. Host-dependent type 1 cytokine responses driven by inactivated viruses may fail to default in the absence of IL-2 or IFN-a/b. J Gen Virol 85, 795-803.

Estrada-Parra S, O Velasco-Castrejon, F Rébora, ML Díaz, J Padierna. 1983. Inmunoterapia de la tuberculosis pulmonar avanzada con factor de transferencia específico. Salud Pública Mex 25, 589-599.

Fabre RA, TM Pérez, LD Aguilar, MJ Range, I Estrada-García, R Hernández-Pando, S Estrada-Parra. 2004. Transfer factors as immunotherapy and supplement of chemotherapy in experimental pulmonary tuberculosis. Clin Exp Immunol 136, 215-223.

Gould MJ. 1998. Limulus Amebocyte Lysate assays and filters applications. In: Meltzer TH, Jornitz MW (eds). Filtration in the Biopharmaceutical Industry. Marcel Dekker, New York, USA, Pp 605-618.

Kirkpatrik CH, RR Rich, TK Smith. 1972. Effects of transfer factor on lymphocyte function in anergicpatients. J Clin Inv 51, 2948-2958.

Klesius PH, JJ Giambrone. 1984. Adoptive transfer of delayed hypersensitivity and protective immunity to Eimeria tenella with chicken-derived transfer factor. Poult Sci 63, 1333-1337.

Kogut MH, L Rothwell, P Kaiser. 2002. Differential effects of age on chicken heterophil functional activation by recombinant chicken interleukin-2. Dev Comp Immunol 26, 817-830.

Kohno K, J Kataoka, T Ohtsuki, Y Suemoto, I Okamoto, M Usui, M Ikeda, M Kurimoto. 1997. IFN-gamma-inducing factor (IGIF) is a costimulatory factor on the activation of Th1 but not Th2 cells and exerts its effect independently of IL-12. J Immunol 158, 1541-1550.

Lawrence HS. 1955. The transfer in humans of delayed skin sensitivity to streptococcal M substance and to tuberculin with disrupted leukocytes. J Clin Invest 34, 219.

Levin AS, VS Byers, HH Fudenberg, J Wybran, AJ Hackett, JO Johnston, LE Spitles. 1975. Osteogenic sarcoma: immunologic parameters before and during immunotherapy with tumor specific transfer factor. J Clin Invest 55, 487-499.

Mateos RA. 1992. Transfer factor immunotherapy in clinically sick lactatibg calves. Vet Mex 23, 4-8.

McCormick AL, S Thomas, W Heath. 2001. Immunization with an interferon- $\gamma$ gp120 fusion protein induces enhanced immune responses to human immunodeficiency virus gp120.J Infect Dis 184, 1423-1430.

Min W, HS Lillehoj, J Burnside, KC Weining, P Staeheli, JJ Zhu. 2001. Adjuvant effects of IL-1beta, IL-2, IL-8, IL-15, IFN-alpha,
IFNgamma, TGF-beta 4 and lymphotaction on DNA vaccination against Eimeria acervulina. Vaccine 20, 267-274.

Ortiz AL, FI Rojas, G Núñez, FA Rodríguez, S Estrada-Parra. 2006. Uso de factor de transferencia como alternativa de tratamiento en lesiones persistentes de infecciones por el virus del papiloma humano (IVPH). Memorias del $1^{\text {er }}$ encuentro internacional sobre factor de transferencia "Sherwood Lawrence", México DF, México.

Padierna L, S Godinez, J Díaz, E García, MA Argaez, O Velasco, J Padierna, S Estrada-Parra. 1985. Factor de transferencia en pacientes con herpes zóster. Infectología 11, 293-299.

Pizza G, F Chiodo, V Colangeli, F Gritti, E Raise, HH Fudenberg, C De Vinci, D Viza. 1996. Preliminary observations using HIV-specific transfer factor in AIDS. Biotherapy 9, 41-47.

Rabinovich NR, P Mclnnes, D Klein, B Fentontlall. 1994. Vaccine Technologies: View to the future. Science 265, 1401-1404.

Robledo-Ávila FH, SM Pérez-Tapia, I Wong-Baeza, A Isibasi-Araujo, I Estrada-García, S Estrada-Parra. 2006. Estudio de la presencia de ligandos para TLR2 y TLR4 en el DLE humano. Memorias del $1^{e r}$ encuentro internacional sobre factor de transferencia "Sherwood Lawrence". México DF, México.

Rojas BS. 1987. Uso de suero hiperinmune y del factor de transferencia para la prevención y tratamiento de la colibacilosis neonatal en lechones. Tesis de licenciatura, Facultad de Medicina Veterinaria y Zootecnia, Universidad Nacional Autónoma de México, México DF, México.

Samuel CE. 2001. Antiviral actions of interferons. Clin Microbiol Rev $14,778-809$.

Schijns VE, C Scholtes, I Zuilekom, E Sanders, L Nicolson, J Argyle. 2002. Facilitation of antibody forming responses to viral vaccine antigens in young cats by recombinant baculovirus-expressed feline IFN- $\gamma$. Vaccine 20, 1718-1724.

Secretaría de Agricultura, Ganadería y Desarrollo Rural. 1995. Norma Oficial Mexicana NOM-033-Z00-1995. Sacrificio humanitario de los animales domésticos y silvestres. Memoria. México DF, México.

Snapper CM, E Paul. 1987. Interferon-gamma and B cell stimulatory factor-1 reciprocally regulates Ig isotype production. Science 236, 944-947.

Spitler LE, AS Levin, DP Stites, HH Fudenberg, B Pirofsky, CS August, ER Stiehm, WH Hitzig, RA Gatti. 1972. The Wiskott-Aldrich syndrome: results of transfer factor therapy. J Clin Inv 51, 3216-24.

Stark GR, M Kerr, RG Williams, H Silverman, D Scheiber. 1998. How cells respond to interferons. Annu Rev Biochem 67, 227-64.

Steele RW, MG Myers, MM Vincent. 1980. Transfer factor for the prevention of varicella-zoster infection in childhood leukemia. N Engl J Med 303, 355-359.

Wilson GB, HH Fudenberg. 1981. Leukocyte migration inhibition as a method for assaying transfer factor activities. Lymphokines Res 4 , 170-173.

Wilson GB. 1988. De Novo initiation of specific cell-mediated immune responsiveness in chickens by transfer factor (specific immunity inducers) obtained from bovine colostrums and milk. Acta Virol 32, 6-18.

Yilma T, S Owens, H Fennie, P Anderson. 1989. Enhancement of primary and secondary immune responses by interferon. Adv Exp Med Biol 251, 145-152. 